\title{
Cardiotoxicity testing of diglycolic acid using In Vitro and In Vivo models
}

Richard Calvert, Miriam E. Mossoba, Keenan Bailey, Howard Toomer, Sanah Vohra, Zachary Keltner, Vanessa Topping, Thomas Black, Nicholas Olejnik, Ana Depina, Kathleen Belgrave, Jessica Sprando, Paddy L. Wiesenfeld and Robert L. Sprando*

*Correspondence: robert.sprando@fda.hhs.gov

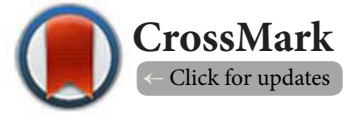

U.S. Food and Drug Administration (US FDA), Center for Food Safety and Applied Nutrition (CFSAN), Office of Applied Research and Safety Assessment (OARSA), Division of Applied Regulatory Toxicology (DART), 8301 Muirkirk Rd., Laurel, MD 20708, USA.

\begin{abstract}
Background: Renal and hepatotoxicity of diglycolic acid (DGA) has been described with in vitro cellular models as well as in vivo animal and human systems. The possibility of DGA being toxic to other organs, such as the heart, has not been well investigated. A human case report identified the heart as a potential target organ of DGA, but an in-house in vivo rat study neither found gross nor microscopic pathological changes following repeated oral DGA exposure.

Methods: To better understand the potential of DGA to adversely affect the heart, we focused our current study on evaluating cardiac effects of DGA by treating rat H9c2 cardiomyocytes and human induced pluripotent stem cells (iPSCs) that were differentiated into beating cardiomyocytes (iCells) with increasing concentrations of DGA in cell culture media. We investigated the effects, mechanisms, and value of our in vitro cellular cardiac models.

Results: We measured mild effects of DGA on cytotoxicity and the cellular production of reactive oxygen species in H9c2 cells. Cardiomyocyte beat rate (BPM) was also mildly affected by transient treatment with DGA.

Conclusions: Our study indicates that DGA cardiotoxicity in vitro correlates with the preliminary findings in our in vivo study where rats treated with daily doses of up to $300 \mathrm{mg} / \mathrm{kg}$ of DGA orally for 5 days did not show any major signs of abnormal cardiac pathology.
\end{abstract}

Keywords: Diglycolic acid, heart, cardiotoxicity, in vitro

\section{Introduction}

Filler or thickener compounds present in dietary supplements or other foods often include carboxymethyl starches, such as carboxymethyl cellulose (CMC) [1]. Interestingly, it has been found that during the chemical synthesis of CMC, a byproduct called diglycolic acid (DGA) is also made and that DGA is a difficult impurity to eliminate $[2,3]$. DGA is a small four-carbon molecule consisting of two carboxylic acids connected together by an ether linkage (PubChem CID: 8088). The safety of DGA as an indirect food additive is the focus of our study. Mass poisoning incidents caused by unintentional ingestion of diethylene glycol have been recently demonstrated to be caused by its metabolic byproduct, DGA [4]. Although the consumption of DGA as a food additive has not been linked to any adverse event, its widespread presence led us to question its potential toxicity. In the absence of published in vivo dose-response and lethal dose (LD)50 data on DGA, we sought out to begin investigating the potential for DGA to engender toxicity. Human in vitro and rat in vivo studies by our group and others have established that DGA is an acute renal and hepatic toxin, with the potential of also being a cardiotoxin [5-10]. In a tragic case report of unintentional human DGA oral exposure, not only were the kidneys and liver severely damaged, but there was some evidence of heart injury as well [9]. Our rat in vivo study, for example, revealed that after daily gavage dosing of different concentrations of DGA ranging up to $300 \mathrm{mg} / \mathrm{kg} \mathrm{BW}$, creatine kinase levels doubled or tripled in the animals that were treated with high doses of DGA, but no direct findings of gross or microscopic cardiac pathology [10]. These preliminary findings may suggest the possibility that the heart is vulnerable 
to the primary or secondary effects of DGA. Furthermore, the possibility of DGA-induced cardiotoxicity is also supported by recent findings that human cardiomyocytes express molecular transporters of organic anions including dicarboxylic acids $[11,12]$. As a dicarboxylic acid, DGA has the ability to chelate divalent cations [13], which could be a mechanism to behave as a possible toxin. Based on these reports therefore, we began an investigation into the potential cardiotoxicity of DGA. Our cardiotoxicity study of DGA aimed to bring together two in vitro cellular models using the established rat-derived cell line, $\mathrm{H} 9 \mathrm{c} 2$, as well as the newer human induced pluripotent cell (iPSC)-derived cardiomyocytes (iCells), which actually beat in vitro. Having achieved its aim, this study contributes to the in vitro toxicity profile of DGA in a cardiac model and even affords us the opportunity to assess the level of concordance between our in vitro rat and human cellular findings with those of our in vivo rat and others' in vivo human data. The strength of any laboratory model of human outcomes will ultimately be contingent upon its ability to predict human clinical data.

\section{Materials and methods \\ Cell culture and treatments}

Two types of cells were used in our study. First type was H9c2 rat cardiomyoblast cell line (ATTC, Manassas, VA), whose cells were initially seeded into T75 flasks and expanded. The initial growth media was DMEM (high glucose) with $4 \mathrm{mM}$ L-glutamine, $110 \mathrm{mg} / \mathrm{L}$ sodium pyruvate, $10 \% \mathrm{FBS}$ and $1 \%$ antibiotic/antimycotic solution. All components were obtained from Life Technologies (Frederick, MD, USA), except for FBS, which was obtained from Thermo-Fisher (Waltham, MA, USA). After propagation in growth media, cells were removed with by gentle treatment with a trypsin solution and plated at $2 \times 10^{5}$ cells per $\mathrm{ml}$ in either white or black 96 -well plates with transparent bottoms. On the day after plating, the cells were differentiated by replacing the growth media with differentiation media. The differentiation media was the same composition as growth media, with $2 \%$ adult goat serum (Thermo-Fisher) replacing FBS and addition of $20 \mathrm{nM}$ each of all-trans and 9-cis retinoic acids. Cells were grown for seven days in differentiation media (changed every two days) prior to use in cell viability assays.

The second type of cellular in vitro model used was iCell cardiomyocytes (Cellular Dynamics, Inc., Madison, WI, USA). Human induced pluripotent stem cell (iPSC) cardiomyocytes (iCell cardiomyocytes) were used to produce a 96-well plate of synchronously beating heart cells. The production of the cells is a proprietary process, but has been described in general terms elsewhere $[14,15]$. Cardiomyocytes were initially seeded at a density of $2 \times 10^{5}$ cells per $\mathrm{ml}$ into gelatin-coated black 96-well plates with transparent bottoms. The different media preparations for plating and subsequent maintenance were provided by Cellular Dynamics. Cells were placed in a humidified air incubator with $5 \% \mathrm{CO}_{2}$ at $37^{\circ} \mathrm{C}$. After $48 \mathrm{~h}$, the surface of the cell layer within the wells was gently washed by slowly aspirating media up and down with a multichannel pipette to suspend non-adherent cells. Plating media was removed, and replaced with warm maintenance media. Maintenance media was changed every other day. Cells were maintained for 10 to 15 days post-plating, which was usually the time required for the cells to beat synchronously in all of the wells.

\section{Cytotoxicity and reactive oxygen species evaluation in H9c2 cells}

H9c2 cells were used to screen the test compounds for overt cytotoxicity, prior to their use in beating cells. Cells were exposed to concentrations of DGA from 0.5 to $10 \mathrm{mM}$ for a 24 hour period, similar to our other studies $[7,8,16,17]$. Cytotoxicity was assessed using a kit for ATP content (Promega, Madison, WI, USA) according to the manufacturer's instructions, except for prior rinsing of wells to avoid interference of the test compounds with light production [16]. Cytotoxicity was also measured in some iCell experiments, after 30 minutes of exposure to DGA at the same range of concentrations.

The production of reactive oxygen species following H9c2 treatment with DGA was assayed using the Promega luminescence system. Following the manufacturer's instructions, cells treated with DGA or Doxorubicin were exposed to a hydrogen peroxide substrate during the last 5 hours of their total treatment ( $24 \mathrm{hrs}$ ) followed by 'Detection Reagent' for approximately 20 minutes. All 96-well assay plates were analyzed by plate reader (BMG Fluorostar by BMG Labtech, Ortenberg, Germany).

\section{Measurement of $\mathrm{pH}$ of media plus DGA}

DGA was obtained from Sigma-Aldrich and was reported to be of $98 \%$ purity. After reading the plates with the FLIPR Tetra instrument to evaluate cell beating (see below), the media was removed and pooled from six wells which received the same concentration of DGA. Media was frozen in a $-80^{\circ} \mathrm{C}$ freezer. About seven days later, it was thawed and heated to $37^{\circ} \mathrm{C}$ in a water bath placed in the cell culture incubator with a $\mathrm{CO}_{2}$ level of 4.9 to $5.0 \%$, simulating the conditions under which the cells were tested. The $\mathrm{pH}$ was measured with a Fisher Orion Star LogR pH meter (Fisher scientific) equipped with a Ross electrode (Fisher).

\section{Beat rate measurement}

Both standard DGA and neutralized DGA were used to study cytotoxicity and effects on beat rate in iCells. A $100 \mathrm{mM}$ aqueous solution of DGA was prepared, then diluted to $0.5 \mathrm{mM}, 1$ $\mathrm{mM}, 2.5 \mathrm{mM}, 5 \mathrm{mM}, 7.5 \mathrm{mM}$, and $10 \mathrm{mM}$ for experimental use. In the case of neutralized DGA, the $100 \mathrm{uM}$ stock was titrated to the $\mathrm{pH}$ of a 50:50 mixture of the media and the EarlyTox calcium detection dye (Molecular Devices) then diluted as above. A FLIPR Tetra instrument (Molecular Devices LLC, Sunnyvale, CA, USA) was used for all measurements with the beating cells. The FLIPR Tetra is a rapid fluorescence plate reader, capable of taking rapid sequential exposures of all wells of a 96 -well 
plate simultaneously. Signal detection was based on changes in fluorescence, measured with an excitation wavelength of 470-495 nm, and an emission wavelength of 515-575 nm. The principle of the instrument is based on the binding of calcium to a fluorescent dye once it enters the cytoplasm from extracellular sources and the sarcoplasmic reticulum. As calcium increases, a parallel increase in fluorescence occurs, which then declines back to baseline with subsequent reduction in intracellular calcium concentration. Calcium movement corresponds to heart cell contraction, each peak representing one beat. Software supplied with the instrument was used to calculate beats per minute (BPM). In the case of very slow beating, the beat rate was determined manually by counting the peaks present over the two minute sampling interval.

After a pre-determined two hour incubation with a calcium binding dye, 96-well plates of beating iCells were placed in the FLIPR Tetra instrument for reading. After baseline readings were obtained, the cells were returned to the incubator for $15 \mathrm{~min}$. Test compounds were then added, utilizing the automated pipette in the FLIPR Tetra to add the compounds to all 96-wells simultaneously. Cells were then returned to the incubator for $30 \mathrm{~min}$, based on use of this exposure interval in a study of iCell cardiomyocytes exposed to pharmaceuticals [18]. Following the 30 min exposure for treatment, plates were read again to measure the effect of the compounds. All experiments were repeated 2 to 3 times.

Because of differing beat rates pre-treatment, all wells were normalized by dividing the post-treatment value by the pretreatment value to yield a ratio. All values from a plate were standardized by division by the mean control value for that plate. Data from all plates were then combined for analysis. This was allowable because of the similarity among standardized means for each treatment on multiple plates. Statistical analysis was done using Microsoft Excel and GraphPad software (GraphPad, Inc.).

\section{Results}

DGA is cytotoxic to H9c2 cardiomyocytes in vitro

To evaluate the potential cytotoxicity of DGA to cardiomyocytes, we exposed H9c2 cells to increasing doses of either DGA or the control cardiotoxin, doxorubicin, for 24 hours in vitro. As expected, doxorubicin treatments lead to a significant decrease in $\mathrm{H} 9 \mathrm{c} 2$ viability starting with the low exposure dose of 0.5 uM ( $P<0.05$, Figure 1a). Relative to this strong toxicity exhibited by doxorubicin, DGA did not significantly compromise cell viability until the exposure dose reached $7.5 \mathrm{mM}(\mathrm{P}<0.05)$, which is a dose 15,000 -fold higher than that of first marked toxic dose of doxorubicin in this series of experiments and likely worked with varying mechanisms to produce these different results.

To corroborate these findings, we next quantified the levels of reactive oxygen species (ROS) in $\mathrm{H} 9 \mathrm{c} 2$ cultures exposed to DGA or doxorubicin. Just as the two compounds produced varying degrees of cytotoxic effects on cardiomyocytes in vitro, they also appeared to exert these effects by different mechanisms of action. In vitro treatment with DGA led to significantly higher bursts of ROS production $(P<0.05)$ at the same treatment concentrations that also significantly decreased H9c2 cell viability (Figure 1b). Doxorubicin, however, did not significantly affect ROS production at any of the concentrations in the 0-10 uM range tested, despite the finding that this whole range of treatment doses strongly reduced cell viability.

DGA cytotoxicicity is not quenched by $\mathrm{pH}$ neutralization We hypothesized that DGA may potentially yield cardiomyocyte toxicity in vitro by virtue of its acidic nature. Although cell culture media is routinely buffered, its buffering capacity may have been exceeded by DGA. As shown in Figure 2a, measuring the $\mathrm{pH}$ value of each DGA treatment concentration in the $0-10 \mathrm{mM}$ range showed that the buffering capacity of cell culture media is indeed exceeded at the $5 \mathrm{mM}$ dose and
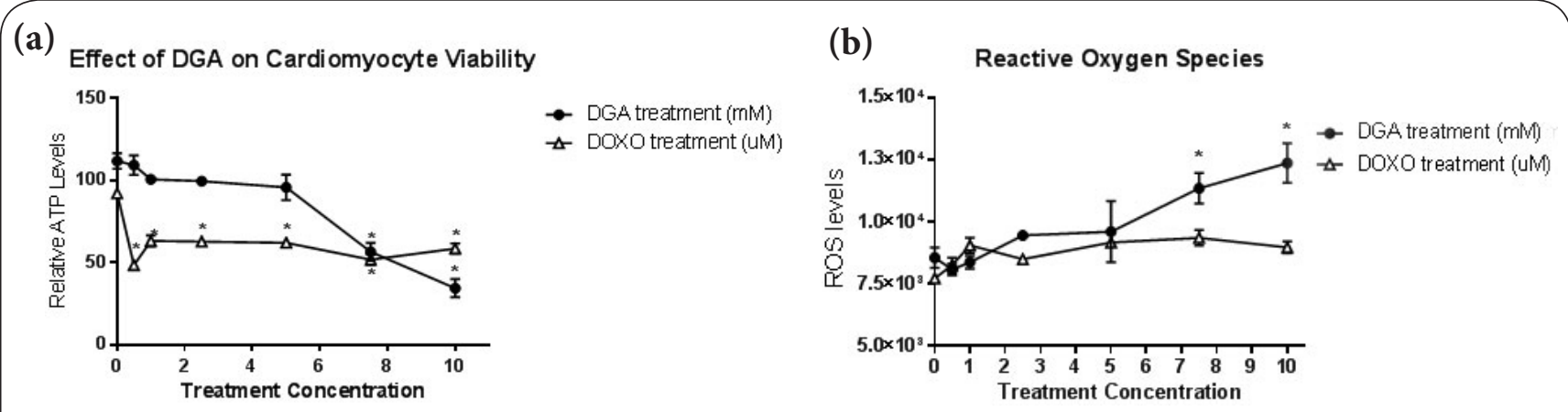

Figure 1. The effect of DGA on cardiomyocyte viability and ROS production. H9c2 cells were exposed for 24 hours to either DGA at concentrations ranging from 0 to $10 \mathrm{mM}$ (filled circles) or doxorubicin at the lower concentration range of 0 to $10 \mathrm{uM}$ (open triangles). (a) Cellular ATP levels of treated cells were normalized to those of untreated cells following assessment by luminescence assay. (b) Cellular ROS levels were also measured by luminescence assay following DGA or doxorubicin treatment. Graphs shown include data from at least 3 independent experiments. ${ }^{\star}, \mathrm{P}<0.05$, DGA ordoxorubicin treatment vs. no treatment. 
Calvert et al., Journal of Toxicology and Health 2017,

beyond $(\mathrm{P}<0.05)$. Interestingly, the $\mathrm{pH}$ values within this treatment testing range remained above 7 .

Given the statistically significant decrease in $\mathrm{pH}$ that DGA could produce in cell culture media, we tested whether neutralizing the acidity of DGA could reduce its cytotoxic potential. Surprisingly, we found that if the $\mathrm{pH}$ levels of DGA-treated cell cultures were kept at the same levels as those of 'no DGA' treatment controls $(\mathrm{pH} \sim 7.8)$, then there was no effect on DGA-induced cytotoxicity. As shown in Figure $\mathbf{2} \mathbf{b}$, the trend in cell viability reduction following treatment with DGA or neutralized DGA were very similar.

Effect of DGA on frequency of beating is pH-dependent Although maintaining the culture media $\mathrm{pH}$ around $7.8 \mathrm{did}$ not appear to affect the ability of DGA to induce cytotoxicity in H9c2 cells, we sought to test whether DGA could instead affect the beating capability of cardiomyocytes. Using iCells as our beating cardiomyocyte in vitro cellular model, we tested this hypothesis. In the absence of $\mathrm{pH}$-neutralizing intervention, we treated iCells with DGA in the treatment dose range of $0-10 \mathrm{mM}$ and measured the frequency of cell beating after 30 minutes of DGA exposure. As a control for beat rate inten- sification, we used $10 \mathrm{uM}$ isoproterenol and as a negative (no effect) control, we used 50 uM amoxicillin [19]. As expected, isoproterenol induced an increase in beating frequency and amoxicillin did not affect beating. By contrast, DGA treatment was associated with a sharp increase in beating at the exposure dose of $2.5 \mathrm{mM}$, followed by a complete cessation of beating over the $2.5 \mathrm{mM}$ dose (Figure 3a). In the presence of neutralized DGA treatments in the same $0-10 \mathrm{mM}$ concentration range, iCell beating frequency increased mildly until cells were exposed to $7.5 \mathrm{mM}$ of neutralized DGA $(P<0.05)$. At 7.5 and $10 \mathrm{mM}$, the frequency of iCell beating was significantly reduced to about $55 \%$ of control (no DGA treatment), as shown in Figure $\mathbf{3 b}(\mathrm{P}<0.05)$.

\section{Discussion}

The identification and characterization of DGA as a renal and hepatic toxicant has been unequivocally established using in vitro cellular, in vivo rat, and even human systems [3,7-10]. A single case report of human exposure indicated that the heart could be negatively affected by DGA, but this effect has never been previously captured in vitro or in vivo animal models. Given the lack of data on the effect of DGA on the

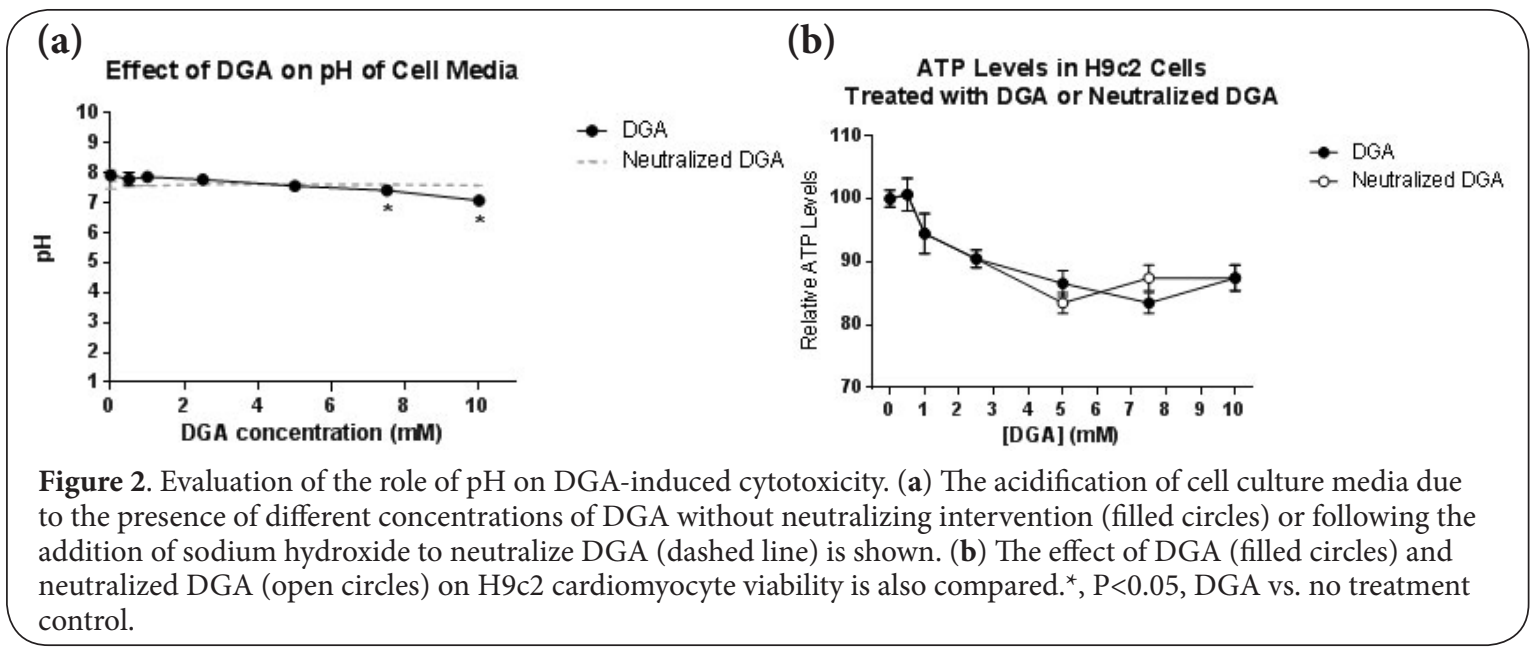

\section{(a)}

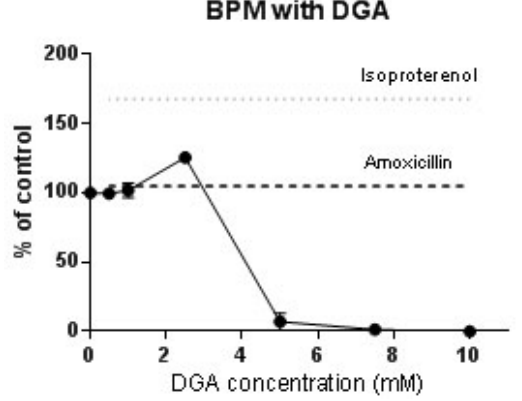

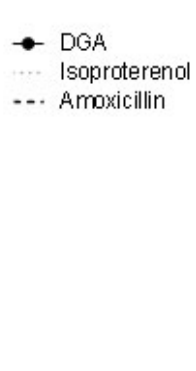

(b)

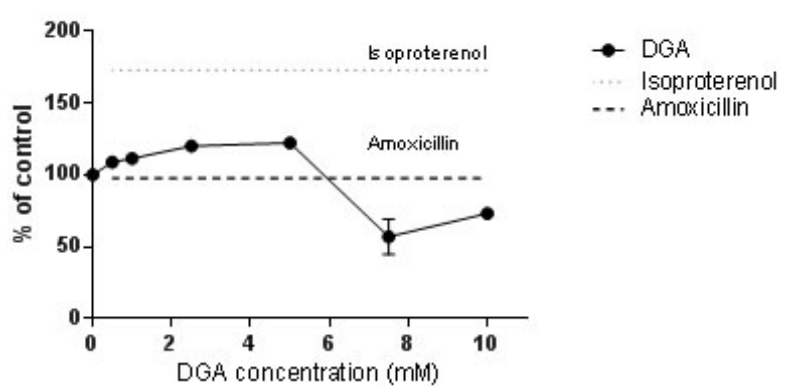

Figure 3. Beat rate of iCells in the presence of DGA. Measurements of beat rate (beats per minute; BPM) were taken following 30 minutes of direct exposure of iCells to DGA in (a) or to neutralized DGA in (b) (filled circle), isoproterenol (dotted gray line), or negative control amoxicillin (dashed black line). ${ }^{*}, \mathrm{P}<0.05, \mathrm{DGA}$ vs. no treatment control. 
heart, we sought to model its potential toxicity using two types of in vitro cellular models. First, for the assessment of cell viability as a fundamental evaluation of cellular toxicity, we used $\mathrm{H} 9 \mathrm{c} 2$ cells, which is a frequently used cardiac in vitro cellular model. The H9c2 cell line is derived from embryonic rat heart tissue and has been validated as a reliable model for its ability to mimic development and disease states [20]. Using this model, we uncovered the potential for DGA to decrease cell viability. This effect was considerably less potent than that of control cardiotoxin doxorubicin and may have worked through a different mechanism of cytotoxicity that involves mitochondrial poisoning through ROS surge production. Although we considered the possibility that the acidifying effect of DGA was primarily responsible for its mode of cytotoxic action, but further investigation challenged this simple concept. By using a second in vitro cellular model using human-derived induced pluripotent stem cell (iPSC), we could generate beating iCell cardiomyocytes in vitro and assess the effect of DGA and its acidity on beat rate. The use of iPSCs is becoming a popular approach to modeling sophisticated heart functions in vitro. The differentiation pathway of iPSCs to beating cardiomyocytes is well established and extremely useful in an in vitro setting. In our experience, the beat rate of cardiomyocytes was not only reduced to zero by DGA past the threshold dose of $5 \mathrm{mM}$, but revealed that the role of DGA's acidic properties was partly responsible for its effect on beat rate. Once the cell culture medium was prepared to have the same $\mathrm{pH}$ as its control medium lacking DGA, we measured attenuation of beat rate reduction. Clearly, features other than DGA's acidity accounted for its apparent beat rate toxicity, since the reduced frequency remained around $50 \%$ following its $\mathrm{pH}$ neutralization. In addition to changes in beating rate, we also observed that DGA could negatively affect $\mathrm{Ca}^{2+}$ signal amplitude of the beating iCells (data not shown), which is consistent with DGA's ability to chelate divalent metal ions as an additional potential mechanism of toxicity as it has been described in the renal system [21].

By investigating the in vitro effects of DGA, we gained the opportunity to compare our findings to that of a recent in vivo rat study that was also recently performed by members of our research group [10]. In that study, rats were orally dosed on a daily basis with DGA dose solution ranging in strength from 0 to $300 \mathrm{mg} / \mathrm{kg}$ BW for a total of up to 30 days. There in, organ weights were measured to help indicate organ abnormalities and it was found that heart weights were not significantly different in any cohort. The levels of creatine kinase, however, were about 2- to 3-fold higher in rats in the two highest dose groups (100 and $300 \mathrm{mg} / \mathrm{kg} \mathrm{BW)} \mathrm{compared} \mathrm{to} \mathrm{levels} \mathrm{in} \mathrm{rats}$ in the vehicle control group. Because creatine kinase activity can reflect both heart and skeletal muscle injury, it is not an exclusive heart-specific biomarker of toxicity, making it difficult to firmly conclude that the measured elevations were due to DGA effects on the heart. However, in light of the case report describing a man who accidentally ingested DGA and was eventually found to have heart problems [9], it might be reasonable to suspect a connection between DGA poisoning and heart disturbances. The reported heart abnormalities included petechiae, slight ventricular hypertrophy, areas of fibrosis, as well as infiltration by interstitial fat, lymphocytes and other cells. It should be noted that the DGA-to-heart connection may also be secondary to the kidney and liver toxicities that appear to be so profoundly injurious. For example, rising levels of serum potassium due to renal dysfunction might have caused the terminal cardiac arrest described in this case report.

\section{Conclusions}

Clearly, the role of DGA in mediating cardiotoxicity has yet to be fully explored, but our study points to the possibility that it may not only compromise cell viability but also affect the beat rate of cardiomyocytes. Reports demonstrating the expression of organic anion transporters on cardiac cells have emerged $[11,12]$ and support the idea that DGA could exert a direct effect on heart cells in addition to possibly affecting heart tissue through secondary mechanisms following renal insufficiency and liver injury. The concordance between the data collected from our in vitro model with our in vivo rat model reflects the potential strength of in vitro systems to forecast in vivo animal model results. The added concordance between these two systems and the unique human case report further supports the utility of both rat and human in vitro cellular model systems in predicting human outcomes of toxin-mediated organ injury.

\section{List of abbreviations}

DGA: Diglycolic acid

iPSCs: Induced pluripotent stem cells

BMP: Beats per minute

\section{Competing interests}

The authors declare that they have no competing interests.

Authors' contributions

\begin{tabular}{|l|c|c|c|c|c|c|c|c|c|c|c|c|c|c|}
\hline Authors' contributions & RC & MEM & KB & HT & SV & ZK & VT & TB & NO & AD & KB & JS & PLW & RLS \\
\hline Research concept and design & $\checkmark$ & $\checkmark$ & -- & -- & -- & -- & -- & -- & -- & -- & -- & -- & $\checkmark$ & $\checkmark$ \\
\hline Collection and/or assembly of data & $\checkmark$ & $\checkmark$ & $\checkmark$ & $\checkmark$ & $\checkmark$ & $\checkmark$ & $\checkmark$ & $\checkmark$ & $\checkmark$ & $\checkmark$ & $\checkmark$ & $\checkmark$ & -- & $\checkmark$ \\
\hline Data analysis and interpretation & $\checkmark$ & $\checkmark$ & -- & -- & -- & -- & -- & -- & -- & -- & -- & -- & -- & $\checkmark$ \\
\hline Writing the article & $\checkmark$ & $\checkmark$ & -- & -- & -- & -- & -- & -- & -- & -- & -- & -- & -- & -- \\
\hline Critical revision of the article & -- & -- & -- & -- & -- & -- & -- & -- & -- & -- & -- & -- & $\checkmark$ & $\checkmark$ \\
\hline Final approval of article & -- & -- & -- & -- & -- & -- & -- & -- & -- & -- & -- & -- & $\checkmark$ & $\checkmark$ \\
\hline Statistical analysis & $\checkmark$ & $\checkmark$ & -- & -- & -- & -- & -- & -- & -- & -- & -- & -- & -- & -- \\
\hline
\end{tabular}


Calvert et al., Journal of Toxicology and Health 2017,

\section{Acknowledgement and funding}

We thank Dr. Thomas Flynn at FDA for helpful discussions.

This work was funded by internal research funds at the FDA.

\section{Publication history}

Editor: Cengiz Gokbulut, Balikesir University, Turkey.

Received: 14-Dec-2016 Final Revised: 17-Mar-2017

Accepted: 28-Apr-2017 Published: 14-May-2017

\section{References}

1. Saha $D$ and Bhattacharya $S$. Hydrocolloids as thickening and gelling agents in food: a critical review. J Food Sci Technol. 2010; 47:587-97. Article | PubMed Abstact | PubMed FullText

2. Koschella A, Hartlieb M and Heinze T. A "click-chemistry" approach to cellulose-based hydrogels. CarbohydrPolym. 2011; 86:154-61. | Article

3. Landry GM, Martin S and McMartin KE. Diglycolic acid is the nephrotoxic metabolite in diethylene glycol poisoning inducing necrosis in human proximal tubule cells in vitro. Toxicol Sci. 2011; 124:35-44. | Article | PubMed

4. Schep LJ, Slaughter RJ, Temple WA and Beasley DM. Diethylene glycol poisoning. Clin Toxicol (Phila). 2009; 47:525-35. | Article | PubMed

5. Besenhofer LM, McLaren MC, Latimer B, Bartels M, Filary MJ, Perala AW and McMartin KE. Role of tissue metabolite accumulation in the renal toxicity of diethylene glycol. Toxicol Sci. 2011; 123:374-83. | Article | PubMed

6. Landry GM, Dunning CL, Conrad T, Hitt MJ and McMartin KE. Diglycolic acid inhibits succinate dehydrogenase activity in human proximal tubule cells leading to mitochondrial dysfunction and cell death. Toxicol Lett. 2013; 221:176-84. | Article | PubMed

7. Mossoba ME, Vohra S, Pugh-Bishop S, Keltner Z, Topping V and Black T et al. In Vitro Exposure of Diglycolic Acid to Human Proximal Tubule Cells Models Acute In Vivo Nephrotoxicity. Manuscript submitted 2017.

8. Mossoba ME, Vohra S, Pugh-Bishop S, Keltner Z, Topping V and Black T et al. Diglycolic Acid Induces HepG2/C3A Liver Cell Toxicity In vitro. Manuscript submitted 2017.

9. Roscher AA, Jussek E, Noguchi T and Franklin S. Fatal accidental diglycolic acid intoxication. Toxicol Pathol. 1975; 3:3-13.

10. Sprando RL, Mossoba ME, Black T, Keltner Z, Vohra S and Olejnik N et al. 28-day Repeated Dose Response Study of Diglycolic Acid in Rats: Renal and Hepatic Effects. Food Chem Toxicol. 2017. I Article I PubMed

11. Nigam SK, Bush KT, Martovetsky G, Ahn SY, Liu HC, Richard E, Bhatnagar $\mathrm{V}$ and Wu W. The organic anion transporter (OAT) family: a systems biology perspective. Physiol Rev. 2015; 95:83-123. | Article | PubMed Abstact | PubMed FullText

12. Roth M, Obaidat A and Hagenbuch B. OATPs, OATs and OCTs: the organic anion and cation transporters of the SLCO and SLC22A gene superfamilies. Br J Pharmacol. 2012; 165:1260-87. | Article | PubMed Abstact | PubMed FullText

13. Motekaitis RJ and Martell AE. New multidentate ligands. Xxv. The coordination chemistry of divalent metal ions with diglycolic acid, carboxymethyltartronic acid and ditartronic acid. J CoordChem. 1984; 13:265-71. | Article

14. Babiarz JE, Ravon M, Sridhar S, Ravindran P, Swanson B, Bitter H, Weiser T, Chiao E, Certa U and Kolaja KL. Determination of the human cardiomyocyte mRNA and miRNA differentiation network by fine-scale profiling. Stem Cells Dev. 2012; 21:1956-65. | Article | PubMed Abstact | PubMed FullText

15. Ma J, Guo L, Fiene SJ, Anson BD, Thomson JA, Kamp TJ, Kolaja KL, Swanson BJ and January CT. High purity human-induced pluripotent stem cell-derived cardiomyocytes: electrophysiological properties of action potentials and ionic currents. Am J Physiol Heart Circ Physiol. 2011; 301:H2006-17. | Article | PubMed Abstact | PubMed FullText

16. Calvert RJ and Vohra S. Doxorubicin-treated H9c2 cells: caution with luminescent ATP and Hoechst 33258 assays. In Vitro Cell Dev Biol Anim. 2013; 49:95-6. | Article I PubMed
17. Calvert R, Vohra S, Ferguson M and Wiesenfeld P. A beating heart cell model to predict cardiotoxicity: effects of the dietary supplement ingredients higenamine, phenylethylamine, ephedrine and caffeine. Food Chem Toxicol. 2015; 78:207-13. | Article | PubMed

18. Sirenko O, Crittenden C, Callamaras N, Hesley J, Chen YW, Funes C, Rusyn I, Anson B and Cromwell EF. Multiparameter in vitro assessment of compound effects on cardiomyocyte physiology using iPSC cells. J Biomol Screen. 2013; 18:39-53. | Article | PubMed

19. Lode H, Janisch P, Kupper $\mathrm{G}$ and Weuta H. Comparative clinical pharmacology of three ampicillins and amoxicillin administered orally. $J$ Infect Dis. 1974; 129:suppl:S156-68. | Article | PubMed

20. Watkins SJ, Borthwick GM and Arthur HM. The H9C2 cell line and primary neonatal cardiomyocyte cells show similar hypertrophic responses in vitro. In Vitro Cell Dev Biol Anim. 2011; 47:125-31. I Article I PubMed

21. Conrad T, Landry GM, Aw TY, Nichols R and McMartin KE. Diglycolic acid, the toxic metabolite of diethylene glycol, chelates calcium and produces renal mitochondrial dysfunction in vitro. Clin Toxicol (Phila). 2016; 54:501-11. | Article | PubMed

\section{Citation:}

Calvert R, Mossoba ME, Bailey K, Toomer H, Vohra S, Keltner Z, Topping V, Black T, Olejnik N, Depina A, Belgrave K, Sprando J, Wiesenfeld PL and Sprando RL. Cardiotoxicity testing of diglycolic acid using In Vitro and In Vivo models. J Toxicol Health. 2017; 4:3. http://dx.doi.org/10.7243/2056-3779-4-3 